\title{
RALPH CUDWORTH Y EL LABERINTO DEL LIBRE ARBITRIO. SUÁREZ, HOBBES Y LEIBNIZ A LA LUZ DE SU TREATISE OF FREEWILL* \\ Griselda Gaiada**
}

RESUMEN: Este artículo aborda el problema del libre arbitrio atendiendo a las relaciones entre el entendimiento y la voluntad. El debate de cuño escolástico acerca de cuál de ambas facultades debe ser considerada libre se prolongó en la modernidad temprana y dio lugar a diferentes respuestas: voluntaristas, intelectualistas y necesitaristas. La primera parte de este trabajo presenta esas tres posiciones a la luz de las doctrinas de Suárez, Leibniz y Hobbes. La segunda se ocupa de las críticas que Ralph Cudworth les opuso en su Treatise of Freewill. Como se verá, cada una de tales soluciones engendra diferentes expresiones de lo que llamaremos la "paradoja de las facultades", que el platónico de Cambridge buscó superar mediante una novedosa psicología de la acción.

\section{sose \\ RALPH CUDWORTH AND THE LABYRINTH OF FREE WILL. SUAREZ, HOBBES AND LEIBNIZ IN THE LIGHT OF HIS TREATISE OF FREEWILL}

ABSTRACT: This article addresses the problem of free will by examining the relationship between the understanding and the will. The scholastic debate about which of both faculties should be considered free was continued in early Modernity, giving rise to different answers: voluntarists, intellectualists and necessitarians. The first part of this paper presents these three positions in the light of the doctrines of Suárez, Leibniz and Hobbes. The second part is devoted to the Ralph Cudworth's criticisms against them in his Treatise of Freewill. As it will be seen, each of these solutions produces different expressions of what I will call the "paradox of the faculties", which the Cambridge Platonist sought to overcome through an original psychology of action.

\footnotetext{
* Este artículo fue realizado en el marco de una beca de nivel superior de la Agencia Nacional de Promoción Científica y Tecnológica de Argentina, obtenida por el proyecto de investigación: "La metafísica modal leibniziana: necesidad y contingencia. Contradicciones y fisuras en el pensamiento de Leibniz". Este último se inscribe dentro del PICT-2017-0506 (ANPCyT).

** CEFHIC-Universidad Nacional de Quilmes.
} 
Se prohíbe su reproducción total o parcial por cualquier medio, incluido electrónico, sin permiso previo y por escrito de los editores.

GRISELDA GAIADA

PALABRAS CLAVE: Entendimiento, intelectualismo, necesitarismo, voluntarismo.

KEY WORDS: Intellectualism, necessitarianism, understanding, voluntarism.

DoI: $10.5347 / 01856383.0138 .000302709$

Estudios 139, pp. 113-134, vol. XIX, invierno 2021. 


\section{RALPH CUDWORTH Y EL LABERINTO DEL LIBRE ARBITRIO. SUÁREZ, HOBBES Y LEIBNIZ A LA LUZ DE SU TREATISE OF FREEWILL}

¿Qué albedrio es el nuestro, si Dios, antes de encender las estrellas, conocía todos nuestros actos y nuestros más recónditos pensamientos? Jorge Luis Borges

Al menos desde san Agustín, la cuestión del liberum arbitrium ha preocupado sin cesar a la filosofía, no solo con relación a la responsabilidad por las acciones voluntarias, sino también en lo que atañe al misterio de la libertad que confronta al hombre con el gran drama de la caída. En torno a la herencia griega del alma y sus principios, se urdió progresivamente una compleja trama en la que la superioridad del principio racional se trabó con conceptos cada vez más alambicados. El intelecto y la voluntad estuvieron en el centro del asunto, por cuanto el concepto de libertad basculó de una facultad a la otra, según la prioridad que se les concediera dentro del alma racional. Así pues, las relaciones entre el entendimiento y la voluntad, lejos de ganar claridad, se vieron oscurecidas por una jerga escolástica cada vez más abstrusa. Los filósofos del siglo XVII, en buena parte herederos de la carga medieval, prestaron singular atención a ambas facultades, prolongando el debate escolástico acerca de dónde correspondía situar la libertad formal de los seres racionales. Suponer que la libertad reside en 
el entendimiento con exclusión de la voluntad, o bien en la voluntad al margen del entendimiento, no fue una cuestión menor, ya que cualquiera de estas opciones compromete el sentido del libre arbitrio.

Como difícilmente pueden encontrarse filósofos que entre los siglos XVI y XVII no se hayan pronunciado al respecto, el propósito inmediato de este trabajo se limita a ofrecer un análisis de las posiciones de Francisco Suárez (1548-1617), Thomas Hobbes (1588-1679) y Gottfried Wilhelm Leibniz (1646-1716). La elección de estos nombres no es casual, sino que se explica por el hecho de que cada uno se asocia a una de las grandes corrientes con las que el problema del libre arbitrio quedaría luego etiquetado: voluntarismo, necesitarismo e intelectualismo. Además de presentar sus puntos de vista, en la segunda sección se ahondará en las críticas que les opuso Ralph Cudworth, cuya agudeza y profundidad filosóficas ameritan un lugar más destacado en la bibliografía especializada. En nuestra opinión, Cudworth puede ser considerado, si no como el juez que dirime la controversia, al menos como uno de los pocos autores modernos que penetró en el corazón del problema del libre arbitrio, mostrando como nadie las deficiencias de los argumentos esgrimidos habitualmente. Él fue quien vislumbró la "paradoja de las facultades",

116 por así llamarla, presente tanto en interpretaciones intelectualistas como voluntaristas del libre arbitrio.

Por lo anterior, el Tratado del libre arbitrio de Cudworth constituirá aquí una pieza clave. Como su título lo indica, tiene por objeto el problema de la libertad y de la responsabilidad moral, además de presentar una novedosa psicología de la acción autónoma que redefine al libre arbitrio como "un poder del alma que combina las funciones del intelecto y la volición"1 (hegemonicon). Pensado probablemente como la tercera parte del True intellectual system of the universe, publicado en vida (1678), no es posible determinar a ciencia cierta por qué Cudworth habría aplazado indefinidamente su publicación. El tratado tiene más bien la forma de un borrador, y ciertamente su contenido se superpone

${ }^{1}$ Sarah Hutton, Introducción a A treatise concerning eternal and immutable morality with a treatise of freewill de Ralph Cudworth, ed. por Sarah Hutton (Cambridge: Cambridge University Press, 1996), 26. 
en gran medida con el de su Sistema y con el de su otro manuscrito póstumo, A treatise concerning eternal and immutable morality, concebido, al parecer, como la segunda parte del libro de 1678.

\section{La relación entre el entendimiento y la voluntad. Si hay libertad formal, ¿en qué facultad del alma reside?}

Como se ha dicho, son tres las corrientes principales que explican la manera en la que diferentes filósofos abordaron el problema del libre arbitrio: 1) el necesitarismo, según el cual el intelecto determina totalmente a la voluntad, de modo que una vez producido el juicio intelectual, la voluntad es incapaz de contradecirlo o, en su ausencia, de poder elegir algo; 2) el intelectualismo, según el cual el intelecto funciona como la razón determinante de la voluntad y su juicio la precede mostrándole el bien que debe seguir; 3) el voluntarismo, según el cual la voluntad determina al acto por sí misma y el juicio del entendimiento no es la causa de su inclinación voluntaria hacia el objeto.

A cada una de estas posiciones subyacen tres preguntas clave, a partir de las cuales analizaremos las corrientes mencionadas. En primer lugar, ¿qué facultad origina la determinación de la voluntad?; esto es, si se trata de una determinación extrínseca o intrínseca. En segundo lugar, ¿qué tipo de relación existe entre el entendimiento y la voluntad? A saber, si el entendimiento es el que mueve a la voluntad a su ejercicio, su juicio la determina necesariamente o no. $\mathrm{O}$ bien, si es la voluntad la que inicia el movimiento del entendimiento para que este despliegue un juicio práctico, ¿cuál es el alcance de este juicio? Por último, ¿en qué facultad reside la libertad formal? Suponiendo que el entendimiento no esté determinado por causas suficientes que vuelvan necesario el "efecto" que se sigue de la elección, ${ }^{2}$ es decir, admitiendo que hay libertad más allá de un sentido negativo (ausencia de obstáculos al movimiento), aparece la pregunta acerca de dónde reside dicha libertad, en la mente o en la voluntad.

${ }^{2}$ Thomas Hobbes, Of liberty and necessity, en Hobbes and Bramhall on liberty and necessity, ed. por Vere Chappell (Cambridge: Cambridge University Press, 1999), § 11, 20-21. 


\section{El necesitarismo de Hobbes}

Hobbes, como se sabe, consideró que la eficacia natural de los objetos determina a los agentes voluntarios, haciendo necesaria no solo su acción, sino también su voluntad. A su juicio, las acciones libres se reducen a simples "efectos" y, al igual que en la legalidad natural, toda acción "voluntaria" está necesitada por las cadenas de causas, cuyo primer eslabón es Dios. Ni el efecto proveniente de la "elección” ni la propia voluntad del agente escapan a la necesidad, puesto que todo lo que ocurre en la deliberación es tan necesario y dependiente de una causa suficiente como cualquier otro hecho del mundo.

Así por ejemplo — dice Hobbes - no es más necesario que el fuego arda que el hecho de que un hombre u otra criatura, cuyos miembros se mueven a su antojo, tenga elección, esto es, la libertad de hacer lo que desea hacer, aunque no esté en su poder o voluntad elegir su deseo, o elegir su elección o voluntad. ${ }^{3}$

Aquí se ve manifiestamente que el libre arbitrio no posee ningún espesor para Hobbes; ni la acción ni el ejercicio de la voluntad estarían en poder del hombre. No obstante, sus reflexiones permiten responder a la pregunta acerca de dónde se sitúa el origen de la determinación de la voluntad. Para él, es el entendimiento el que determina o, mejor dicho, "necesita" la acción que se sigue de la voluntad (determinación extrínseca), aunque su juicio no constituya toda la causa suficiente, ${ }^{4}$ sino la última parte de ella, como sucede cuando se añade una última pluma que hace peso suficiente para quebrar la espalda del animal de carga. ${ }^{5} \mathrm{La}$ crítica a las palabras de Medea (video meliora proboque, deteriora sequor) que le opuso Bramhall son elocuentes al respecto: Medea vio muchas razones, nos dice Hobbes, para abstenerse de matar a sus hijos, pero el último juicio de su entendimiento no fue otro que tomarlos como objeto

${ }^{3}$ Ibid., $\S 11,21$. Salvo que se indique lo contrario, todas las traducciones son mías.

${ }^{4}$ Ibid., $\S 31,38$.

${ }^{5}$ Ibid., $\S 23,34$. 
de venganza contra su marido, ${ }^{6}$ de modo que la acción malvada se siguió necesariamente. ${ }^{7}$

La voluntad se reduce entonces al "último apetito" que antecede a la acción, y su función no es otra que traducir en acto el "pensamiento actual" que en cierto momento tiene el agente. Así, por ejemplo, en situación de ira, la acción seguirá el "pensamiento de venganza", o en situación de miedo súbito, el "pensamiento de escape". ${ }^{8}$ En esta perspectiva necesitarista, la voluntad carece, pues, de jurisdicción para ejercer una verdadera causación libre (voluntariedad intrínseca). A decir verdad, extremando la posición de Hobbes, el "último apetito" de la voluntad apenas si difiere del "último pensamiento" que precede a la acción, rozando muy de cerca la paradoja de un intelecto que quiere necesariamente. Si la absoluta necesidad natural se aplica también a las acciones o "efectos particulares" provenientes de un agente - llamado "voluntario"

${ }^{6}$ Cabe señalar aquí que Leibniz utilizó la famosa frase de Medea en su Confessio philosophi (1672/1673?) en un sentido que no dista demasiado del hobbesiano, al menos en lo que concierne a la determinación que opera el entendimiento sobre la voluntad. Aunque no llegue al extremo de un entendimiento que "necesita" el acto de la voluntad, Leibniz interpreta la frase de Medea a la luz del alcance de un entendimiento finito: no es que Medea piense que es lo peor, sino que el entendimiento juzga la venganza como un bien mayor que el mal del crimen (A VI 3, 134-135). En este periodo, se constata la ausencia de una teorización de la voluntad como una facultad autónoma, por lo que su competencia no parece ser otra que seguir el mandato intelectual. En este sentido, Yakira destaca la dificultad que sobreviene en tal contexto: "lo que resulta difícil de entender es cómo la voluntad, que por su propia esencia es únicamente determinable por algo diferente de ella, puede seguir siendo considerada libre". Elhanan Yakira, "El problema de la libertad humana en Leibniz y Spinoza", en Leibniz frente a Spinoza. Una interpretación panorámica, ed. por Leticia Cabañas et al. (Granada: Comares, 2014), 219.

${ }^{7}$ Suárez aventuró en sus Disputaciones metafísicas (1597) este tipo de respuesta, aunque confesó no encontrarla en ningún autor en particular de su época. Creyó que era posible argumentar de manera plausible que "la voluntad está completamente determinada a su elección por el juicio del intelecto, de modo tal que 1) en ausencia de ese juicio, la voluntad no puede estar determinada a elegir nada, y que 2) una vez que el juicio ha sido dado, la voluntad es incapaz de estar en desacuerdo con el mismo — esto es, incapaz de no conformarse a dicho juicio-". Así pues, una vez que se ha producido el juicio de la razón, "1) la voluntad no está en poder de no actuar (si ha sido juzgado que debe actuar), o bien 2) no está en su poder actuar (si ha sido juzgado que no debe actuar)". Desde luego, Suárez consideró inaceptable que la voluntad se comporte "pasivamente antes que activamente" y que se la someta al "movimiento de otra facultad por una especie de necesidad". Francisco Suárez, On efficient causality: Metaphysical disputations 17, 18, and 19, trad. por Alfred J. Freddoso (New Haven: Yale University Press, 1994), Disp. 19, Sec. $5, \S 12,336$.

${ }^{8}$ Hobbes, Of liberty and necessity, $\S 25,37$. 
solo por no interrumpir en vida su deliberación — ${ }^{9}$ ningún lugar podría serle atribuido a la "libertad formal".

\section{El intelectualismo de Leibniz}

A diferencia de Hobbes, Leibniz rechazó que la necesidad física pudiera afectar a las almas. Como exigencia de su armonía prestablecida, las causas libres difieren de las causas naturales: no solo no son subsumibles bajo la legalidad natural, sino que además son espontáneas. La espontaneidad, en rigor atribuible a toda sustancia simple por ser principio de acción, debe ser entendida aquí como el "imperio" que tiene una sustancia inteligente sobre sus acciones, ${ }^{10}$ es decir, como la libertad de los seres racionales. En la Teodicea leemos que "la inteligencia es como el alma de la libertad", ${ }^{11}$ frase que bien podría constituir el leitmotiv del "intelectualismo" que comúnmente se ha atribuido a Leibniz. Ya en De libertate a necessitate in eligendo (1680-1684?), el filósofo había afirmado que "la mente no está determinada por algo externo, sino por sí misma", de modo que seguiría siendo activa, aun cuando toda otra cosa (excepto Dios) dejara de existir. Por ende, "en esta potencia [que tiene la mente] de determinarse a sí misma para actuar consiste la naturaleza de la libertad". ${ }^{12}$

Esto nos adelanta, en principio, la respuesta acerca de dónde situar la raíz de la libertad formal, que Leibniz formuló explícitamente en $D e$ libertate et gratia (1680-1684?) como sigue: "la libertad tiene su raíz en la naturaleza de la mente, porque solamente las mentes son libres". ${ }^{13}$ En su opinión, la mente se inclina por razones verdaderas o aparentes al bien, pero nunca puede darse una inclinación tan fuerte que haga que el acto se siga necesariamente. ${ }^{14}$ Dicho de otro modo, siempre es capaz

${ }^{9}$ Ibid., $\S 28,38$.

${ }^{10}$ Leibniz, Teodicea, § 291, GP VI, 289.

${ }^{11} \mathrm{Ibid}$ \$ 288, GP VI, 288.

${ }^{12}$ Leibniz, Confessio philosophi, A VI 4, 1451: "Et in hac potentia seipsam determinandi ad agendum consistit natura libertatis."

${ }^{13} \mathrm{Ibid}$., 4, 1455: "Libertas ita explicanda est ut radicem suam habeat in natura mentis quia solae mentes sunt liberae."

${ }^{14}$ Leibniz insistió contra John Wyclif y Hobbes en que la libertad que se encuentra en las mentes está exenta tanto de coacción como de necesidad. Véase: ibid, 1452. 
de suspender el juicio, porque ninguna apariencia del bien puede ser tan determinante como para que ello no suceda. ${ }^{15}$ Así pues, la "mente elige"16 en función de lo que delibera, pero "nada habrá de elegir"17 en ausencia de la deliberación. El libre arbitrio parece ser exclusivo patrimonio intelectual, y difícilmente se podría dudar del intelectualismo que Leibniz habría abrazado. Por otra parte, la pregunta acerca de qué facultad inicia la determinación de la voluntad tampoco parece dar lugar a dudas: la voluntad no haría más que seguir las razones que le muestra el entendimiento. No solo este último determinaría a la facultad apetitiva - cuya voluntariedad no sabría no ser extrínseca-, sino que, además, al menos en cierta selección de textos, la voluntad se vería impedida de oponer un acto contrario al juicio intelectual. $\mathrm{Su}$ pasividad sería tal que no haría más que querer el último juicio del entendimiento práctico.

Sin embargo, la relación entre el entendimiento y la voluntad se complica al considerar otras afirmaciones de Leibniz, como, por ejemplo, cuando dice que "la relación entre el juicio y la voluntad no es tan necesaria como podría pensarse". ${ }^{18}$ En la Teodicea, se afirma que la esencia de la voluntad es "el esfuerzo de actuar tras el juicio". No obstante, como hace falta tiempo para que este esfuerzo llegue a su cima, puede ocurrir que se lo suspenda o modifique por la intervención de nuevas percepciones o inclinaciones, capaces de hacer que la mente se desvíe o forme un juicio contrario. ${ }^{19}$ Además, agrega Leibniz, "tampoco seguimos siempre el último juicio del entendimiento práctico, determinándonos a quererlo", ${ }^{20}$ ya que a menudo el entendimiento procede por "pensamientos sordos" que no alcanzan a conmover lo suficiente a la voluntad. ${ }^{21}$ Por ende, habría que admitir que la voluntad puede permanecer insensible o incluso rechazar el juicio expreso del entendimiento, si apetece lo contrario.

${ }^{15}$ Ibid., 4, 1456.

${ }^{16}$ Ibid.: "Mens eligit".

${ }^{17} \mathrm{Ibid}$., 4, 1457: "Quod si sine deliberatione divertenda est, habemus intentum, scitur enim mentem nihil esse electuram".

${ }^{18}$ Leibniz, Teodicea, § 311, GP VI, 301.

${ }^{19} \mathrm{Ibid}$.

${ }^{20}$ Ibid., § 51, GP VI, 130.

${ }^{21}$ Ibid. $\S 311$, GP VI, 301. 
Al parecer, Leibniz habría matizado en el periodo tardío la pasividad de la voluntad, o más bien, allí asomaría un conato de "voluntariedad intrínseca", que dificulta afirmar sin más que el origen de la determinación de la voluntad proviene siempre del entendimiento. Más aún, surgiría de esto una paradoja: si el entendimiento mueve a la voluntad hacia su ejercicio y objeto, entonces ¿cómo podría esta no estar determinada siempre por el juicio de aquel, esclarecido o no? Y si la voluntad puede determinarse por sí misma en cuanto a su ejercicio y objeto, ¿por qué necesitaría del juicio previo del entendimiento? La respuesta de que la raíz de la libertad está en la mente no soluciona la cuestión, pues reproduce a su manera la paradoja en cuestión: si el entendimiento es la facultad formalmente libre, entonces la voluntad no elige por su cuenta, sino dirigida siempre por lo que la mente elige (voluntariedad extrínseca). Y si la voluntad es capaz de elegir por sí misma, entonces ya no requiere el comando expreso del entendimiento (voluntariedad intrínseca). Por tanto, habrá que reconocerla como una "facultad arbitraria"22 o formalmente libre. Como se advierte, la relación entre ambas facultades no se resuelve meramente en términos intelectualistas. Es cierto que suele prevalecer la idea de que la voluntad precisa de la tutela de la inteligencia para determinarse según el bien, pero también es cierto que Leibniz no la redujo a una "facultad ciega" cuyo objeto le sería dado en préstamo desde afuera. ${ }^{23}$

\section{El voluntarismo suareciano}

Francisco Suárez dedicó buena parte de su Disputación XIX (1597) a responder a la pregunta acerca de qué facultad del alma racional amerita ser considerada formalmente libre. Para ello, analizó cuidadosamente ambas facultades: entendimiento y voluntad. En primer lugar, rechazó cualquier sombra de necesitarismo, ya que a su juicio es absurdo que

${ }^{22}$ La expresión "facultad arbitraria” debe entenderse aquí en su acepción más lata de una facultad que es capaz de elegir, que detenta el libre arbitrio.

${ }^{23}$ Véase: Leibniz, Teodicea, $\S 309-311$, GP VI, 299-301; Conversatio cum Domino Episcopo Stenonio de Libertate, A VI 4, 1375 y 1380. 
una facultad libre con respecto a su ejercicio pueda estar determinada a un acto "por la sola fuerza de la naturaleza". ${ }^{24}$ Por el contrario, su determinación ha de ser voluntaria. Ahora bien, esta determinación voluntaria puede ser de dos clases: 1) o bien la facultad está determinada extrínsecamente mediante un acto voluntario suscitado por otra facultad, 2) o bien lo está mediante un acto intrínsecamente voluntario. E1 primer disyuntivo afirma la "voluntariedad extrínseca" de la voluntad; el segundo, su "voluntariedad intrínseca". En el primer caso, la voluntad no es libre por sí misma, dado que "no se mueve a sí misma con respecto a su ejercicio", 25 sino que es libre como una facultad "comandada" por el entendimiento. En el segundo caso, sí lo es, pues es “capaz de determinarse a sí misma con respecto al ejercicio de un acto libre", ${ }^{26}$ sin necesidad de que el entendimiento la mueva a actuar. Como el entendimiento no es una facultad intrínsecamente voluntaria, concluye Suárez, solo la voluntad puede ser considerada como una facultad formalmente libre.

En cuanto a la pregunta acerca de qué facultad inicia la determinación, el jesuita cree que no puede ser otra que la propia voluntad. Esta no solo se autodetermina, sino que además debe determinar al intelecto para que le ofrezca un juicio práctico respecto del objeto apetecido. El acto intelectual no se produce libremente por obra de la propia inteligencia práctica, sino que requiere "un comando o movimiento de parte de la voluntad que quiere”. Y así, continúa Suárez, "toda la libertad de dicho acto intelectual pertenece formalmente a la voluntad y no al entendimiento". ${ }^{27}$ En otros términos, el origen de la determinación reside en la voluntad, la cual, en virtud de lo que apetece, mueve al entendimiento para que con su acto despliegue un juicio, del que se dice que "la precede", aunque sin determinarla, ya que a ella corresponde determinarse por su entera cuenta. Suárez lo sostiene con diversos argumentos, extraídos del sentido común, de la autoridad de la Iglesia y de las Escrituras. Así, por ejemplo, si se le preguntara a alguien por qué piensa

${ }^{24}$ Suárez, On efficient causality, Disp. 19, Sec. 5, § 19, 340.

${ }^{25}$ Ibid.

${ }^{26}$ Ibid.

${ }^{27}$ Ibid., Sec. 5, § 20, 341. 
en cierta cosa más bien que en otra, dirá que "lo hace porque quiere". 28 También se prueba por la opinión de los teólogos, quienes, a partir de las Escrituras (1 Corintios, 7:37), consideraron que toda bondad o maldad se asienta en la voluntad. Es por eso que a menudo, nos dice Suárez, los Antiguos Padres llamaron a la libre elección simplemente "voluntad libre". ${ }^{29}$

Además, sostener lo contrario, esto es, que el intelecto es formalmente libre con respecto al ejercicio de su acto, acarrea el problema de una duplicación del mal. La argumentación de Suárez discurre así: tan pronto como el intelecto juzgara que cierto objeto malo es susceptible de ser elegido, habría maldad en el acto intelectual considerado en sí mismo y antes del acto de consentimiento de la voluntad. La atribución indebida de una libertad que no le pertenece conduciría al absurdo de un intelecto que disputa consigo mismo, o se contradice a sí mismo, rechazando el mandato del bien que brota de sí (puesto que no habría propiamente error, sino culpa). A su vez, cuando la voluntad realiza el acto de elegir dicho objeto, se incurriría nuevamente en maldad, en la medida en que su acto es evitable y contrario a la razón. ${ }^{30}$ Más aún, mientras la voluntad no prestara su consentimiento, pero permaneciera el juicio intelectual, la persona merecería ser reprendida por el juicio fallido del

124 intelecto libre. Para Suárez, todo esto es absurdo, pues no puede haber pecado allí donde no hay nada de voluntario. ${ }^{31}$ En suma, el pecado no puede ser diferido a una operación que antecede al acto mismo de querer pecar. Solo si velit, peccare possit.

Así pues, el intelecto no hace más que brindar la regla práctica a la voluntad, lo que se explica por la naturaleza de esta última, que para Suárez "es, por así decirlo, ciega". ${ }^{32}$ No obstante tal cosa, como la voluntad tiende siempre al objeto mediante "una inclinación perfecta y voluntaria", "es capaz de libertad, y en esto incluso excedería en cierto sentido al intelecto". ${ }^{33}$ Como defensor de un sentido renovador de

${ }^{28}$ Ibid., Sec. 5, § 20, 340.

${ }^{29}$ Ibid., Sec. 5, § 24, 343-344.

${ }^{30}$ Ibid., Sec. 5, § 25, 344.

${ }^{31}$ Ibid.

${ }^{32}$ Ibid., Sec. 5, § 25, 345.

${ }^{33}$ Ibid. 
la contingencia, ${ }^{34}$ el jesuita hizo de la voluntad una facultad autodeterminante, es decir, plenamente capaz de determinarse o no por la regla intelectual.

\section{Críticas de Cudworth a las tres posiciones. El principio hegemónico del alma}

\section{Contra el necesitarismo}

Debido a lo polémica que fue la figura de Hobbes entre los miembros de la Royal Society y entre los así llamados platónicos de Cambridge, Cudworth dedicó buena parte de su Tratado del libre arbitrio a analizar los dichos de Hobbes relativos a la necesidad. Ya el primer capítulo apunta de lleno a socavar el necesitarismo hobbesiano. Su argumento se centra en el ateísmo al que conduciría la filosofía de Hobbes. Si se aceptase que las acciones humanas son necesarias, nos dice Cudworth, no solo se horadaría la distinción entre el mal de culpa y los males necesarios, sino también la idea misma de justicia vindicativa y desde luego la creencia en el juicio final. Sus críticas se desarrollan en cuatro argumentos que retoman los ataques de Hobbes a la atribución de una libertad contingente al hombre. ${ }^{35}$ Aquí solo nos ocuparemos del último argumento, por ser el que representa el nervio del necesitarismo.

${ }^{34} \mathrm{Al}$ respecto, véase Griselda Gaiada, "Sobre el tablero de la libertad: La partida entre la contingencia y la determinación. Leibniz y Lady Conway contra Molina y Suárez", Revista Latinoamericana de Filosofía 41, núm. 1 (2015): 103-119.

${ }^{35}$ No vamos a entrar aquí en el detalle de las críticas que Cudworth dirige contra Hobbes por fundamentar la necesidad de la agencia humana en la naturaleza de Dios, el único al que en rigor correspondería la libertad contingente. Solo diremos que Cudworth rechaza que la libertad contingente de la voluntad humana atente contra la presciencia divina. A su criterio, la ignorancia en lógica de Hobbes no tiene parangón, ya que confunde la necesidad de la proposición disyuntiva con la necesidad de sus disyuntivos: "[Hobbes] dice que si hay necesidad en la disyunción, debe haberla en una u otra de sus dos partes tomadas aisladamente. Si no hay necesidad de que algo suceda, entonces debe ser necesario que no suceda, como si no pudiera haber necesidad en la disyunción, aunque ambos miembros de la misma sean contingentes y ninguno de ellos necesario. Esto es una ignorancia vergonzosa en lógica, especialmente para quien se jacta de hacer demostraciones geométricas." Cudworth, A treatise of freewill, cap. 22, 203-204. 
Cudworth abre fuego contra Hobbes presentando como sigue su principio según el cual todas nuestras voliciones son necesarias:

Toda volición está determinada por la razón del bien, o la apariencia del bien mayor; ahora bien, las apariencias y razones del bien están en el entendimiento, y en consecuencia no son arbitrarias, sino necesarias, de donde se sigue que todas las voliciones deben ser necesarias. ${ }^{36}$

Como se ve, el pasaje evoca el vínculo que habría para Hobbes entre el entendimiento y la voluntad. En opinión de Cudworth, quienes conciben tal relación en términos de un intelecto que actúa necesariamente sobre sus objetos, sin nada de voluntad que lo determine a su ejercicio o a la especificación del objeto, creen que la voluntad es "ciega" y que el intelecto se conforma a la necesidad de una "cadena de pensamientos" ${ }^{37}$ que la determina por completo. Así pues, sostienen que "la facultad ciega de la voluntad siempre sigue necesariamente el último juicio práctico del entendimiento necesario". ${ }^{38}$ Esto no es otra cosa que afirmar que toda volición y acción humanas están necesitadas, ya que en un contexto así, los apetitos, impulsos y deseos de la voluntad no significan nada, no son más que las apreciaciones y juicios de un entendimiento que actúa en todo necesariamente. ${ }^{39}$

Ahora bien, aun suponiendo que el bien aparente se encontrara en el entendimiento, tampoco se seguiría que "los hombres fuesen meramente pasivos con respecto a las apariencias del bien y a sus propios juicios prácticos". ${ }^{40}$ Para justificarlo, Cudworth apela a Aristóteles, quien ya había rechazado que tales apariencias fuesen necesarias: las apariencias del bien y los juicios prácticos difieren en nosotros según nuestro grado de deliberación y hábitos electivos, es decir, según la manera en que apuntalemos nuestras decisiones a fin de resistir a las

${ }^{36}$ Ibid., cap. 2, 158.

${ }^{37}$ Clara alusión al Leviatán. Véase Thomas Hobbes, Leviatán: La materia forma y poder de un Estado eclesiástico y civil, trad. por Carlos Mellizo (Barcelona: Altaya, 1997), cap. 3, 32.

${ }^{38}$ Cudworth, A treatise of freewill, cap. 5, 168.

${ }^{39}$ Véase: ibid., cap. 6, 169.

${ }^{40}$ Ibid., cap. 21, 203. 
pasiones y "apariencias inferiores" (lower appearances). Por ello, sucede a menudo que los mismos motivos y razones no tienen el mismo efecto en diferentes personas ni tampoco en la misma persona en diferentes momentos de su vida. ${ }^{41}$ De donde hay que concluir que la necesidad de las acciones humanas es un "error vulgar" que ignora la naturaleza humana y el amplio legado de la filosofía antigua.

\section{Contra el intelectualismo}

Cudworth analiza también las dificultades que minan las posiciones intelectualistas. Aunque sus críticas no mencionen a nadie en particular, es lógico suponer que se dirigían principalmente a los partidarios del punto de vista tomista, para quienes "la voluntad sigue las directivas del intelecto". ${ }^{42} \mathrm{Si}$ bien la cronología impide que Leibniz haya sido uno de sus blancos, las objeciones cudworthianas al intelectualismo tienen amplio alcance entre los defensores de la subordinación de la voluntad a la razón, y en ese sentido se las puede aplicar también a las posteriores reflexiones de Leibniz.

Según Cudworth, el problema del libre arbitrio conduce a un bivium o encrucijada entre dos caminos, causada en gran medida por la asimilación de la "jerga escolástica". Intelectualismo y voluntarismo representan, claro está, una y otra vía. En un caso, se entroniza al intelecto como "el iniciador y primer motor de todas las acciones", ${ }^{43}$ pues ignoti nulla cupido. A él corresponde juzgar sobre todas las cosas, no solo sobre la verdad o falsedad de las cosas especulativas, sino también sobre "la elegibilidad de las cosas prácticas", esto es, "sobre lo que debe hacerse o no". ${ }^{44}$ La otra vía, por su parte, inviste a la voluntad del poder de mover al entendimiento y de determinarlo a su ejercicio y especificación del objeto. Es la posición de los defensores de la libertad de indiferencia, de la que trataremos en la última sección.

${ }^{41}$ Ibid., 202-203.

${ }^{42}$ Hutton, "Introducción" en ibid., 26.

${ }^{43}$ Cudworth, A treatise of freewill, cap. 5, 167.

${ }^{44}$ Ibid. 
Lo importante que hay que destacar ahora es que, a juicio de Cudworth, ambos caminos desembocan en una forma de razonar absurda:

Puesto que atribuir el acto de intelección y percepción a la facultad del entendimiento, y los actos de volición a la facultad de la voluntad, o decir que es el entendimiento el que entiende y la voluntad la que quiere, es lo mismo que si se dijera que la facultad de caminar camina, y que la facultad de hablar habla, o bien que la facultad musical interpreta una lección de laúd, o canta tal o cual tema. ${ }^{45}$

La precipitada adopción de la "jerga escolástica” llevaría así a una discriminación artificial de las facultades que ignora el verdadero funcionamiento del alma. Los filósofos, nos dice, admiten generalmente el principio de que las actiones sunt suppositorum. El problema es que muchos de ellos no llegan a advertir que el lenguaje adoptado introduce artificialmente dos supposita, dos cosas subsistentes, dos agentes y dos personas en el alma racional, a las que dan el nombre de entendimiento y voluntad. La forma de hablar de los escolásticos acarrea pues una "confusión inextricable" y deriva en un "absurdo ininteligible", en la medida en que es imposible entender cómo incumbiría solo al entendimiento la mera intelección, sin nada de voluntad, y a la voluntad, la mera volición, sin nada de intelección. ${ }^{46}$ Suponer un entendimiento que prescinde de toda volición, o bien una voluntad que carece de toda intelección, desnaturaliza los poderes o facultades que hay en el alma racional. Así pues, dicha atribución ficticia de competencias deviene causa de múltiples paradojas en las que caen los filósofos.

Desde el punto de vista intelectualista, la "paradoja de las facultades" implicaría para Cudworth una duplicación inútil de funciones: si el entendimiento juzga sobre el bien y debe comandar a la voluntad para que siga su juicio, entonces la voluntad deviene facultad ciega e incapaz de juzgar sobre el bien. El bien aparente o apetecido pasaría pues a la esfera del intelecto con la consiguiente carga volitiva. Pero si se acepta que el bien es objeto de la voluntad, entonces no podría verse cómo ella sería incapaz de reconocerlo mediante su propio ejercicio, con la

${ }^{45}$ Ibid., cap. 7, 170.

${ }^{46}$ Ibid., 171. 
consiguiente carga intelectiva de su lado. Lo mismo ocurre desde el punto de vista de la locación de la libertad formal: si el intelecto es la facultad arbitraria, entonces la voluntad no podría serlo, por lo que no elegiría por su cuenta, sino por lo que la mente ya ha elegido. Pero si se admite que la voluntad es capaz de elegir por sí misma, entonces posee arbitrio, y no podría verse por qué el juicio del entendimiento debería precederla y conducirla siempre al ejercicio de su acto.

La paradoja es manifiesta, y desde luego se la encuentra también al contemplar juntas ciertas afirmaciones dispersas de Leibniz en torno al libre arbitrio. $\mathrm{Si}$ "es cierto e infalible que la mente se determina hacia el máximo bien aparente, y que ningún ejemplo contrario jamás ha sido dado, donde el error, o al menos la irreflexión, no precediera al pecado", ${ }^{47}$ habría que reconocer que el juicio sobre el bien aparente corresponde solo al entendimiento y que la voluntad no hace sino acatar pasivamente ese juicio que la precede. Pero si "la voluntad tiende al bien en general y debe ir hacia la perfección que nos conviene", ${ }^{48}$ por cuanto "voluntatis objectum esse bonum apparens", ${ }^{49}$ deberíamos admitir que puede reconocer el bien por su propia cuenta y determinarse a seguirlo, sin que la exclusividad del juicio recaiga en otra facultad. Según esto, el dilema acerca de si la voluntad es extrínseca o intrínsecamente voluntaria se revela como falso y pone sobre el tapete la paradoja en cuestión, derivada del artificio de un intelecto que solo juzga y de una voluntad que solo quiere. Y es a esto a lo que Cudworth opuso su tesis de la unidad psicológica de la acción, sin negar la diversidad de poderes o facultades que tiene el alma racional:

Es el hombre o el alma que entiende, y el hombre o el alma que quiere, como es el hombre que camina y el hombre que habla o pronuncia un discurso, y como es el músico que interpreta una pieza en el laúd. Por ende, es una y la misma cosa subsistente, una y la misma alma que juzga y quiere a

${ }^{47}$ Leibniz, De libertate a necessitate in eligendo (1680-1684?), A VI 4, 1450: "Videtur dici posse: certum et infallibile esse, ut mens se determinet ad maximum bonum apparens; neque ullum exemplum contrarium unquam fuisse datum, ubi non error peccatum praecesserit, vel saltem incogitantia, ut in peccato primi hominis apparet, qui credebat se usu pomi fore Deo similem."

${ }^{48}$ Leibniz, Teodicea, $\S 33$, GP VI, 122.

${ }^{49}$ Leibniz, Conversatio cum Domino Episcopo Stenonio de Libertate (1677), A VI 4, 1380. 
la vez, y es el mismo agente que actúa de diversas maneras. Y así se puede concebir fácilmente que una y la misma alma racional en nosotros pueda a la vez querer entendiendo o conociendo lo que quiere; y entender o pensar en tal o cual objeto queriéndolo. ${ }^{50}$

\section{Contra el voluntarismo}

La vía voluntarista del bivium desemboca, como se ha visto, en una voluntad que se determina a sí misma con indiferencia de lo que prescribe el entendimiento. Aunque Cudworth no asocie sus reflexiones a nadie en particular, es claro que "tenía en mente a Suárez y a sus seguidores". ${ }^{51}$ Sus críticas se dividen en tres argumentos: el primero concierne a las alternativas de la voluntad, el segundo cuestiona la definición suareciana del libre arbitrio y el último revela la "paradoja de las facultades" desde el punto de vista voluntarista.

En cuanto a lo primero, Cudworth rechaza que la "libertad contingente de autodeterminación" - supuesta la igualdad perfecta de los objetos y la autodeterminación fortuita de la voluntad - pueda constituir el verdadero sentido del libre arbitrio que sirve de fundamento a recompensas y castigos. Si hubiera dos objetos con la misma apariencia, nos dice, nadie podría ser reprendido o premiado por inclinarse a uno antes que al otro. A su juicio, no solo la hipótesis de la igualdad perfecta de las opciones es moralmente improbable — como si el bien y el mal fuesen dos guineas idénticas - , sino que además toda condenación justa solo puede provenir de un acto de desprecio por lo mejor a favor de lo peor allí donde "los objetos son en sí mismos realmente diferentes". ${ }^{52}$ Por lo tanto, nuestra libertad solo puede probarse allí donde el bien y el mal están a nuestro alcance, allí donde habla nuestra conciencia para acusarnos o excusarnos por nuestras decisiones. ${ }^{53}$

El segundo punto de la crítica de Cudworth se dirige al corazón de la doctrina suareciana de la libertad, según la cual "el hombre tiene libre

${ }^{50} \mathrm{Cudworth}$, A treatise of freewill, cap. 7, 171.

${ }^{51}$ Hutton, "Introducción", en ibid., 26.

${ }^{52}$ Cudworth, A treatise of freewill, cap. 5, 166.

${ }^{53}$ Ibid., 167. 
arbitrio, porque puede actuar y no actuar, dados todos los requisitos para actuar". ${ }^{54}$ Si esto ocurre gracias a la voluntad — donde el jesuita ha situado la libertad formal - , pronto se ve que la función del intelecto deviene estéril, puesto que su juicio, denuncia Cudworth, debe estar incluido entre los requisitos dados para actuar. ${ }^{55}$ Dicho de otro modo, si la voluntad puede tanto actuar como no actuar tras haberse producido el juicio intelectual, es como si nunca hubiera existido ese juicio. El problema es, por así decirlo, la gratuidad del movimiento de "abajo arriba" y de "arriba abajo" que defienden los partidarios de la libertad de indiferencia. ¿Qué sentido tiene, pues, que la voluntad inicie la determinación del entendimiento para que este le proponga un juicio práctico, si a fin de cuentas hará lo que quiere con absoluta indiferencia respecto de ese juicio? Las palabras del filósofo son elocuentes:

Hay quienes con el fin de salvar el fenómeno de la libertad de la voluntad, piensan que es necesario suponer, primero que todo, que la voluntad, aunque ciega, determina así y todo al entendimiento, tanto al ejercicio como a la especificación de su objeto. Y pese a que el entendimiento, siendo necesario en sus juicios, solo propone a la voluntad ciega lo que piensa que debe hacerse, o su último juicio práctico en el caso concernido, y nada más, lo hace solamente para atraer e invitar a la voluntad a ello. Pero esta soberana reina o emperatriz del alma, la voluntad ciega, permanece tan libre e indiferente de hacer o no hacer esto o aquello, como si el entendimiento no hubiera dado ningún juicio al respecto, y finalmente se autodetermina de modo fortuito sin consideración alguna del mismo. Tal es el significado de la definición de la libertad que se da comúnmente, a saber: Voluntas, positis omnibus ad agendum requisitis, potest agere, vel non agere. ${ }^{56}$

El pasaje ya anuncia la última crítica, dirigida al problema de la relación entre ambas facultades en lo que concierne al inicio de la determinación. Bajo un aspecto voluntarista que invierte la prioridad de la

${ }^{54}$ Suárez, Disp. 19, Sec. 5, § 4: "Item homo est liberi arbitrii, quia potest operari et non operari, positis omnibus requisitis ad agendum".

${ }^{55}$ Cudworth, A treatise of freewill, cap. 5, 168.

${ }^{56}$ Ibid. 
determinación, reaparece entonces la paradoja en cuestión. Si la voluntad mueve y determina al entendimiento tanto a su ejercicio como a sus objetos, porque precisa el juicio intelectual, entonces no puede explicarse su indiferencia ante este juicio, la cual, claro está, lo tornaría innecesario. Además, si la voluntad es una facultad ciega, no podría, como tal, mover al entendimiento respecto de las opciones que se le presentan. Por último, su ceguera le impediría determinarse a sí misma por lo mejor o lo peor, de modo que carecería de toda responsabilidad moral. A la luz de esto, no podría sostenerse, como lo hace Suárez, que "no hay en el hombre otra bondad o maldad formal y moral que aquella que proviene de la voluntad". ${ }^{57}$ Por otra parte, si se admite que la voluntad es capaz de reconocer la regla del bien que le ofrece el entendimiento, pero de determinarse en su contra si así lo desea, entonces ya no sería una mera facultad ciega sin discernimiento. Habida cuenta de todo esto, Cudworth habló de un círculo sin fin:

Además, [tales filósofos] están forzados a correr alrededor de un círculo sin fin: sostienen que la voluntad no puede querer nada que no le haya sido representado primero por el entendimiento (pues, de otro modo, la voluntad debería querer lo que no sabe qué), y por otra parte que el entendimiento no puede actuar sobre esto o aquello sin ser movido y determinado a ello por la voluntad, de modo que debe haber una acción del entendimiento que precede a todo acto de la voluntad y a la vez un acto de la voluntad que precede a todo acto del entendimiento, lo cual es aún más contradictorio e imposible. ${ }^{58}$

Este círculo sin fin de una voluntad anteponiéndose al entendimiento y de un entendimiento anteponiéndose a la voluntad denuncia para Cudworth los límites de la forma de pensar suareciana, por su desconocimiento de la unidad del alma racional, capaz de querer entendiendo y de entender queriendo. La falla en el argumento indiferentista se explica por dos razones derivadas de la concepción misma de la voluntad. Por un lado, el movimiento de determinación que ejerce la voluntad sobre

${ }^{57}$ Suárez, On efficient causality, Disp. 19, Sec. 5, § 25.

${ }^{58} \mathrm{Cudworth}$, A treatise of freewill, cap. 6, 169. 
el intelecto sería el producto de una acción ciega que no sabe "qué mueve ni por qué lo hace". ${ }^{59}$ Por otro lado, el entendimiento no podría determinar a la voluntad - debido a su naturaleza autodeterminantede modo que esta obrará, en cualquier caso, de manera fortuita y con independencia de los dictados de aquel. El juicio de Cudworth tiene, pues, ecos leibnizianos: "así, esta libertad de querer será mera irracionalidad y locura, actuando o determinando ella misma todas las acciones humanas". ${ }^{60}$ De donde se sigue la inaceptable conclusión de que el hombre querría siempre ignorando las razones, por lo que toda deliberación, todo juicio esclarecido, todo consejo prudente, más aún la razón misma, serían inútiles. A decir verdad, no habría stricto sensu ni virtud ni vicio, y tendría que decirse que quien ha obrado bien lo ha hecho de causalidad y, en caso contrario, que su voluntad ha actuado jure suo. ${ }^{61}$

Ahora bien, la paradoja de las facultades se resuelve —o más bien se la elude - si se evita asumir un discurso escolástico que toma a la voluntad indiferente, esto es, a la irracionalidad, como el principio hegemónico del alma. ${ }^{62}$ Para Cudworth, el principio dominante en el hombre no puede ser identificado con una facultad indiferente a los grados del bien y del mal, que sería "mero ímpetu, fuerza o actividad" ${ }^{63}$ Por el contrario, lo hegemónico en todo hombre es el alma comprendiéndose a sí misma, sosteniéndose a sí misma, como si estuviera en su propia mano (as it were in its own hand), como si estuviera replegada sobre sí (as it were redoubled upon itself), disponiendo de la capacidad para determinarse por lo mejor o lo peor. ${ }^{64}$

Así pues, este hegemonicon determina siempre la capacidad pasiva de la naturaleza del hombre en uno u otro modo, ya sea por lo mejor o por lo peor. Y tiene un poder autoformante y autoestructurante (self-forming and self-framing power), por el cual todo hombre se hace a sí mismo (every

\footnotetext{
${ }^{59}$ Ibid., cap. 8, 172.

${ }^{60}$ Ibid., cap. 6, 169.

${ }^{61}$ Ibid., 170.

${ }^{62}$ Ibid., cap. 9, 177.

${ }^{63}$ Ibid.

${ }^{64}$ Ibid., cap. 10, 178.
} 
man is self-made) lo que es. Y conforme a ello merece recompensa o reprobación, premio o castigo. ${ }^{65}$

A modo de conclusión, cabe decir que este poder autoedificante del alma racional se inserta en un orden ontológico donde la sustancia incorpórea, como principio de actividad, es el fundamento metafísico de lo que sucede a nivel físico. La vida plástica de la naturaleza exhibe sus grados según se trate de una actividad más o menos consciente, más o menos reflexiva. ${ }^{6}$ En este contexto, puede apreciarse el situs ontológico que corresponde a los seres racionales y el lugar que ocupa la libertad para Cudworth. El poder que tiene el hombre sobre sí mismo para deliberar en mayor o menor medida con respecto a la elegibilidad de carácter moral es la raíz de la libertad. Y cuando moldea su alma desplegando la bondad que hay en su centro, el hombre así autoedificado forja su libertad, la libertad de autodeterminarse según lo mejor. ${ }^{67}$ La división artificial de las facultades del alma, a la que suscriben las vertientes intelectualistas, necesitaristas y voluntaristas, impide ver que este poder no se ejerce unilateralmente, ni necesariamente ni indiferentemente. Frente al laberinto de la libertad, Cudworth creyó encontrar una salida situando al libre arbitrio en la plasticidad del alma racional para crear su "lado 134 interior" conforme al amor de Dios.

${ }^{65}$ Ibid.

${ }^{66}$ Sobre este tema, véase: Charles Zarka, Liberté et nécessité chez Hobbes et ses contemporains: Descartes, Cudworth, Spinoza, Leibniz (París: Vrin, 2012), 67-79.

${ }^{67}$ Por el contrario, cuando el principio hegemónico del alma se alía con los apetitos inferiores, experimenta un "poder autodegradante" (self-impairing power) por el cual el hombre deviene causa de sus propios males. Cudworth, A treatise of freewill, cap. 10, 178. 